\title{
Reproductive System Findings Link Identifier
}

National Cancer Institute

\section{Source}

National Cancer Institute. Reproductive System Findings Link Identifier. NCI Thesaurus.

Code C162024.

A sequence of characters used as a linkage between related reproductive system findings identifier records. 